\title{
Comparative architectures of mammalian and chicken genomes reveal highly variable rates of genomic rearrangements across different lineages
}

\author{
Guillaume Bourque, ${ }^{1,5}$ Evgeny M. Zdobnov, ${ }^{2}$ Peer Bork, ${ }^{2}$ Pavel A. Pevzner, ${ }^{3}$ \\ and Glenn Tesler ${ }^{4}$ \\ ${ }^{1}$ Genome Institute of Singapore, Singapore 138672, Republic of Singapore; ${ }^{2}$ European Molecular Biology Laboratory, 69117 \\ Heidelberg, Germany; ${ }^{3}$ Department of Computer Science and Engineering, ${ }^{4}$ Department of Mathematics, University of California, \\ San Diego, La Jolla, California 92093, USA
}

\begin{abstract}
Molecular evolution studies are usually based on the analysis of individual genes and thus reflect only small-range variations in genomic sequences. A complementary approach is to study the evolutionary history of rearrangements in entire genomes based on the analysis of gene orders. The progress in whole genome sequencing provides an unprecedented level of detailed sequence data to infer genome rearrangements through comparative approaches. The comparative analysis of recently sequenced rodent genomes with the human genome revealed evidence for a larger number of rearrangements than previously thought and led to the reconstruction of the putative genomic architecture of the murid rodent ancestor, while the architecture of the ancestral mammalian genome and the rate of rearrangements in the human lineage remained unknown. Sequencing the chicken genome provides an opportunity to reconstruct the architecture of the ancestral mammalian genome by using chicken as an outgroup. Our analysis reveals a very low rate of rearrangements and, in particular, interchromosomal rearrangements in chicken, in the early mammalian ancestor, or in both. The suggested number of interchromosomal rearrangements between the mammalian ancestor and chicken, during an estimated 500 million years of evolution, only slightly exceeds the number of interchromosomal rearrangements that happened in the mouse lineage, over the course of about 87 million years.
\end{abstract}

[Supplemental material is available online at www.genome.org.]

Whole genome sequencing provides an unprecedented level of detailed sequence data for comparative studying of genome organizations beyond the level of individual genes, and highlighting rearrangements shaping our genomes. The analysis revealed evidence for a larger number of rearrangements than previously thought, and shed some light on previously unknown features of eukaryotic evolution (Lander et al. 2001). The comparative analysis of recently sequenced rodent genomes and the human genome allowed reconstruction of the putative genomic architecture of the murid rodent ancestor (Bourque et al. 2004), while the architecture of the ancestral mammalian genome and the rate of rearrangements in the human lineage remained unknown.

Genome rearrangement studies start with identification of corresponding orthologous regions in different genomes. The definition of orthologous regions has been gradually shifting from requirements of DNA-level alignment, corresponding to strict conservation of gene order and orientation and applicable to genomes as close as human and mouse (Lander et al. 2001; Waterston et al. 2002), to a more fuzzy definition accommodating small-range rearrangements to capture intuitively the same gene neighborhood in more diverged genomes (Zdobnov et al. 2002; Kent et al. 2003; Pevzner and Tesler 2003). Once the orthologous regions, termed synteny blocks, are defined, we can explore the space of possible shufflings of the blocks to find a

${ }^{5}$ Corresponding author.

E-mail bourque@gis.a-star.edu.sg; fax 6564789058.

Article and publication are at http://www.genome.org/cgi/doi/10.1101/ gr.3002305. Article published online before print in December 2004. most parsimonious scenario of rearrangements that explains the current organization of the genomes.

Rearrangement analyses were initially restricted to unichromosomal genomes (Palmer and Herbon 1988; Sankoff et al. 1992; Bafna and Pevzner 1995; Blanchette et al. 1999; Cosner et al. 2000). But the advent of large-scale sequencing and comparative mapping projects (O'Brien et al. 1999; Murphy et al. 2000; Lander et al. 2001; Waterston et al. 2002; Gibbs et al. 2004) has encouraged the development of methods to also analyze rearrangements in multichromosomal genomes (Hannenhalli and Pevzner 1995; Bourque and Pevzner 2002; Tesler 2002a).

In the current study, we considered two different types of evidence to establish orthologous genomic regions, referred to in the manuscript as synteny blocks. One stems from DNA-DNA alignments, referred as sequence-based data, and the other from protein-protein alignments, referred to as gene-based data. We then applied GRIMM-Synteny (Pevzner and Tesler 2003) to determine the synteny blocks. These synteny blocks were used as input to the GRIMM (Tesler 2002a,b) and MGR (Bourque and Pevzner 2002) algorithms to reconstruct the likely rearrangement evolutionary scenarios, considering inversions, translocations, fusions, and fissions. In the process, MGR also estimates the number of rearrangement events on the evolutionary tree and suggests the reconstructed genomes of a murid rodent ancestor, referred later as $R A$, and a boreoeutherian ancestor (Murphy et al. 2001) of human, mouse, and rat; the latter is a good approximation to the mammalian ancestor, and therefore we refer to it as $M A$. 


\section{Results}

The analysis of rearrangements in human, mouse, rat, and chicken was done on two distinct data sets. The first data set consisted of gene-based data while the second was obtained directly from sequence-based data; see Methods for further details, and Figure 1 for an illustration. As expected, the results highlight advantages and disadvantages of the two types of inputs, but overall the high level of similarity between the solutions confirms the robustness of the method.

\section{Gene-based data}

To generate synteny blocks using genes as anchors, we started from a set of 6447 four-way orthologous genes, pre-filtered for evidence of conserved pairwise synteny using SyntQL (Zdobnov et al. 2002; E. Zdobnov, unpubl.); see the Methods section. We imposed a minimum of at least three genes per synteny block and allowed for various levels of tolerance for microrearrangements; see the Methods section for a description. The results are shown in Table 1 (see also Supplemental material). The solutions obtained in the various runs are very consistent both in terms of the number of rearrangements on the edges of the recovered scenario (see Table 2) and of the observed chromosomal associations in the putative ancestors (see Table 3 ). In the rest of this section, we present a detailed description of the results on run gene7, which allows for genes to be merged into the same block when there are up to two intervening genes per species. In this run, there are 586 synteny blocks, comprising a total of 6140 genes. Since some of the three-way human-mouse-rat synteny blocks described in Gibbs et al. (2004) and Bourque et al. (2004) have less than three genes, these blocks did not make it into our set of four-way synteny blocks. Furthermore, since not every mammalian gene has an ortholog in chicken, some of the previously defined humanmouse-rat synteny blocks "lost" some of their genes and were deleted in the four-way comparison by falling below the three genes per block requirement. Finally, some of the previously identified three-way blocks were fragmented onto multiple chicken chromosomes or rearranged in chicken, thus splitting them into multiple four-way blocks. As a result, our 586 four-way synteny blocks correspond to only 299 three-way synteny blocks after projecting to human, mouse, and rat genomes. The average size of the four-way synteny blocks is $3.2 \mathrm{Mb}$ in human, $2.9 \mathrm{Mb}$ in rat, $2.8 \mathrm{Mb}$ in mouse, and only $1.2 \mathrm{Mb}$ in chicken.

Using these 586 four-way blocks, GRIMM reveals evidence of at least 441 pairwise rearrangements (of the order and orientation of the whole blocks) between chicken and human, 511 between chicken and mouse, and 506 between chicken and rat. Using the same blocks suggests at least 219 rearrangements between human and mouse, 220 between human and rat, and 75 between mouse and rat. The fact that these last three numbers are $\sim 25 \%$ smaller than the ones described in Bourque et al. (2004) (they were, respectively, 293, 299 are 100) is largely explained by the $-25 \%$ reduction in the number of blocks due to the inclusion of a fourth genome and to the use of genes instead of similarity anchors.

Running MGR on the 586 synteny blocks generates a rearrangement scenario, and two putative ancestors shown in Figure 2. MGR uses heuristics to attempt to minimize the number of rearrangements on the tree, but does not guarantee the result is a most parsimonious solution. On the recovered tree, there are 73 rearrangements between human and $M A$ and 389 between
A)
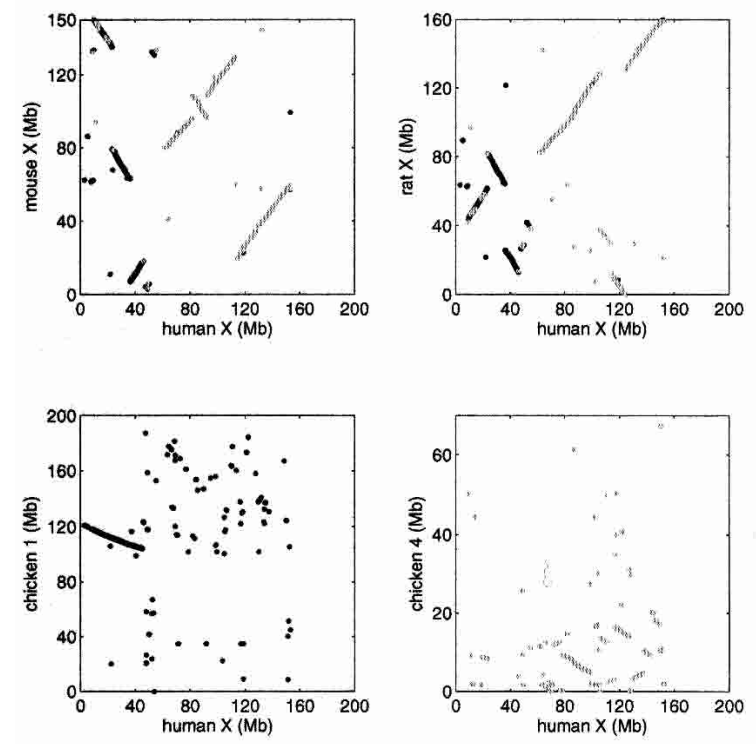

B)
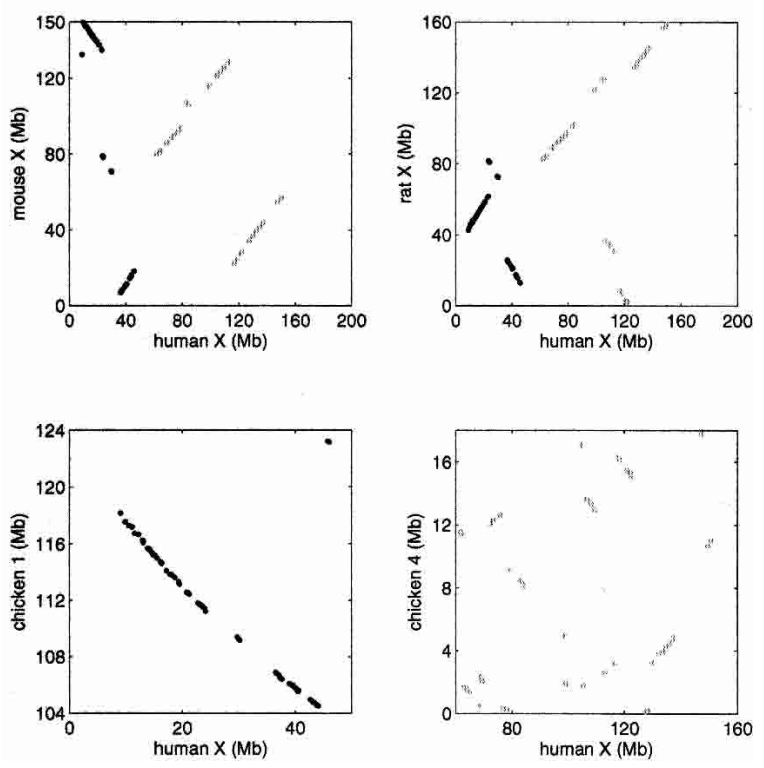

Figure 1. (A) Dot-plots of anchors based on UCSC alignments for the mammalian X-chromosome versus chicken Chromosomes 1 and 4 . The data are very noisy. Many spurious hits are visible on the plots, and there are additional spurious hits between the mammalian X-chromosome and the other chicken chromosomes (data not shown). These spurious hits were filtered out by GRIMM-Synteny because they did not congeal into a block of sufficiently large size. (B) Dot-plots of genes on the mammalian $\mathrm{X}$-chromosome versus chicken Chromosomes 1 and 4 . The data are more sparse but much cleaner than the alignment-based data. There were no spurious hits between the mammalian X-chromosome and the other chicken chromosomes.

chicken and $M A$. There are also 38 rearrangements between mouse and $R A$ and 41 between rat and $R A$. Finally, there are 122 rearrangements between $M A$ and $R A$. These numbers are lower bounds based on the assumption that the tree is correct; it is possible that nature used a less efficient sequence of steps or 
Table 1. Four-way human-mouse-rat-chicken synteny blocks based on orthologous genes

\begin{tabular}{|c|c|c|c|c|}
\hline & Human & Mouse & Rat & Chicken \\
\hline $\begin{array}{l}\text { Chromosomes included } \\
\text { Total length }(\mathrm{Mb})\end{array}$ & $\begin{array}{c}1-22, x \\
3020\end{array}$ & $\begin{array}{l}1-19, x \\
2577\end{array}$ & $\begin{array}{c}1-20, x \\
2720\end{array}$ & $\begin{array}{c}1-24,26-28,32 \mathrm{~W}, \mathrm{Z} \\
940\end{array}$ \\
\hline $\begin{array}{l}\text { Run gene4 } \\
\text { No. of blocks } \\
\text { Mean synteny block length }(\mathrm{kb}) \\
\text { Max synteny block length }(\mathrm{kb}) \\
\text { Total synteny block length }(\mathrm{kb}) \\
\text { Total synteny block length }(\% \text { of genome) } \\
\text { No. of breakpoint regions } \\
\text { Mean breakpoint region length }(\mathrm{kb}) \\
\text { Max breakpoint region length }(\mathrm{kb}) \\
\text { Total breakpoint region length }(\mathrm{kb}) \\
\text { Total breakpoint region length (\% of genome) } \\
\text { No. of genes per block }\end{array}$ & $\begin{array}{c}627 \\
2865 \\
49,242 \\
1,796,071 \\
59.48 \% \\
604 \\
1727 \\
45,875 \\
1,043,037 \\
34.54 \% \\
3-120, \text { mean } 9.7\end{array}$ & $\begin{array}{c}627 \\
2515 \\
45,201 \\
1,576,879 \\
61.18 \% \\
607 \\
1436 \\
25,984 \\
871,671 \\
33.82 \%\end{array}$ & $\begin{array}{c}627 \\
2644 \\
48,457 \\
1,657,662 \\
60.95 \% \\
606 \\
1613 \\
30,583 \\
977,759 \\
35.95 \%\end{array}$ & $\begin{array}{c}627 \\
1075 \\
22,013 \\
673,793 \\
71.67 \% \\
596 \\
410 \\
5725 \\
244,635 \\
26.02 \%\end{array}$ \\
\hline $\begin{array}{l}\text { Run gene7 } \\
\text { No. of blocks } \\
\text { Mean synteny block length }(\mathrm{kb}) \\
\text { Max synteny block length }(\mathrm{kb}) \\
\text { Total synteny block length }(\mathrm{kb}) \\
\text { Total synteny block length }(\% \text { of genome) } \\
\text { No. of breakpoint regions } \\
\text { Mean breakpoint region length }(\mathrm{kb}) \\
\text { Max breakpoint region length }(\mathrm{kb}) \\
\text { Total breakpoint region length }(\mathrm{kb}) \\
\text { Total breakpoint region length (\% of genome) } \\
\text { No. of genes per block }\end{array}$ & $\begin{array}{c}586 \\
3205 \\
49,242 \\
1,877,940 \\
62.19 \% \\
563 \\
1731 \\
30,387 \\
974,336 \\
32.26 \% \\
3-120, \text { mean } 10.5\end{array}$ & $\begin{array}{c}586 \\
2794 \\
45,201 \\
1,637,099 \\
63.52 \% \\
566 \\
1436 \\
18,196 \\
812,924 \\
31.54 \%\end{array}$ & $\begin{array}{c}586 \\
2945 \\
48,457 \\
1,725,810 \\
63.45 \% \\
565 \\
1635 \\
30,583 \\
923,608 \\
33.96 \%\end{array}$ & $\begin{array}{c}586 \\
1196 \\
22,013 \\
700,581 \\
74.52 \% \\
555 \\
394 \\
5725 \\
218,520 \\
23.24 \%\end{array}$ \\
\hline $\begin{array}{l}\text { Run gene } 10 \\
\text { No. of blocks } \\
\text { Mean synteny block length }(\mathrm{kb}) \\
\text { Max snyteny block length }(\mathrm{kb}) \\
\text { Total synteny block length }(\mathrm{kb}) \\
\text { Total synteny block length }(\% \text { of genome) } \\
\text { No. of breakpoint regions } \\
\text { Mean breakpoint region length }(\mathrm{kb}) \\
\text { Max breakpoint region length }(\mathrm{kb}) \\
\text { Total breakpoint region length }(\mathrm{kb}) \\
\text { Total breakpoint region length (\% of genome) } \\
\text { No. of genes per block }\end{array}$ & $\begin{array}{c}567 \\
3358 \\
49,242 \\
1,903,906 \\
63.05 \% \\
544 \\
1745 \\
30,387 \\
949,037 \\
31.43 \% \\
3-120, \text { mean } 10.9\end{array}$ & $\begin{array}{c}567 \\
2930 \\
45,201 \\
1,661,039 \\
64.45 \% \\
547 \\
1442 \\
18,196 \\
788,984 \\
30.61 \%\end{array}$ & $\begin{array}{c}567 \\
3089 \\
48,457 \\
1,751,281 \\
64.39 \% \\
546 \\
1646 \\
30,583 \\
898,578 \\
33.04 \%\end{array}$ & $\begin{array}{c}567 \\
1253 \\
22,013 \\
710,366 \\
75.56 \% \\
536 \\
390 \\
5725 \\
208,834 \\
22.21 \%\end{array}$ \\
\hline $\begin{array}{l}\text { Run gene20 } \\
\text { No. of blocks } \\
\text { Mean synteny block length }(\mathrm{kb}) \\
\text { Max synteny block length }(\mathrm{kb}) \\
\text { Total synteny block length }(\mathrm{kb}) \\
\text { Total synteny block length }(\% \text { of genome) } \\
\text { No. of breakpoint regions } \\
\text { Mean breakpoint region length }(\mathrm{kb}) \\
\text { Max breakpoint region length }(\mathrm{kb}) \\
\text { Total breakpoint region length }(\mathrm{kb}) \\
\text { Total breakpoint region length (\% of genome) } \\
\text { No. of genes per block }\end{array}$ & $\begin{array}{c}518 \\
3787 \\
49,242 \\
1,961,447 \\
64.95 \% \\
495 \\
1801 \\
30,387 \\
891,495 \\
29.52 \% \\
3-120 \text { mean } 12.0\end{array}$ & $\begin{array}{c}518 \\
3327 \\
45,201 \\
1,723,141 \\
66.86 \% \\
498 \\
1460 \\
18,196 \\
726,883 \\
28.20 \%\end{array}$ & $\begin{array}{c}518 \\
3495 \\
48,457 \\
1,810,421 \\
66.56 \% \\
497 \\
1689 \\
30,583 \\
839,439 \\
30.86 \%\end{array}$ & $\begin{array}{c}518 \\
1413 \\
22,013 \\
731,745 \\
77.83 \% \\
487 \\
392 \\
5725 \\
191,143 \\
20.33 \%\end{array}$ \\
\hline
\end{tabular}

Several sets of four-way synteny blocks were formed from orthologous genes by allowing for various levels of rearrangements of the genes within the blocks. Runs gene 4 through gene20 allow an increasing span of rearrangements, as explained in the text under "GRIMM-Synteny parameters." Statistics are shown on the sizes of the blocks and the breakpoint regions between them (excluding chromosome ends).

operations besides those considered. ${ }^{6}$ These numbers, normalized using the MA-human edge, are displayed in Table 2.

The rearrangement scenario corresponding to Figure 2 confirms a high ratio of intrachromosomal versus interchromosomal rearrangements on the chicken edge. This was computed by attempting to maximize the number of inversions on the $M A-$ chicken edge while staying within the constraint of 389 steps;

${ }^{6}$ This is especially true for long edges. An alternative approach would be to use a statistical model (Larget et al. 2002), but that would require a different set of assumptions and lead to different drawbacks. however, it is only an approximation, because (1) there could be an alternative sequence of 389 steps with a higher ratio, and (2) intrachromosomal rearrangements could have been mimicked by interchromosomal rearrangements. This ratio varies: 2.8 on the $M A$-chicken edge, 1.7 on the $R A$-rat edge, 1.4 on $M A$-human edge, 0.7 on the $R A$-mouse edge, and 0.7 on the $M A-R A$ edge. ${ }^{7}$ The number of interchromosomal rearrangements on

${ }^{7}$ Although these ratios depend on the level of microrearrangements tolerated, we found the ratio on the $M A$-chicken edge to be consistently higher in all runs as compared to the one on other edges.

\section{Genome Research}

www.genome.org 
Genome rearrangements in human/mouse/rat/chicken

Table 2. Number of rearrangements on derived evolutionary trees

\begin{tabular}{|c|c|c|c|c|c|c|c|c|c|c|c|c|c|c|}
\hline \multirow[b]{3}{*}{ Run } & \multicolumn{7}{|c|}{ All rearrangements } & \multicolumn{7}{|c|}{ Interchromosomal } \\
\hline & \multicolumn{4}{|c|}{ Gene-base (geneX) } & \multicolumn{3}{|c|}{ Sequence-based } & \multicolumn{4}{|c|}{ Gene-based (geneX) } & \multicolumn{3}{|c|}{ Sequence-based } \\
\hline & 4 & 7 & 10 & 20 & $100 \mathrm{~K}$ & $200 \mathrm{~K}$ & $300 \mathrm{~K}$ & 4 & 7 & 10 & 20 & $100 \mathrm{~K}$ & $200 K$ & $300 K$ \\
\hline MA-chicken & 4.2 & 5.3 & 4.4 & 4.7 & 3.9 & 3.7 & 4.9 & 2.5 & 3.4 & 2.7 & 3.4 & 2.3 & 2.1 & 3.7 \\
\hline$R A$-mouse & 0.4 & 0.5 & 0.5 & 0.5 & 0.5 & 0.5 & 0.7 & 0.6 & 0.8 & 0.7 & 0.8 & 0.5 & 0.5 & 0.7 \\
\hline$R A$-rat & 0.5 & 0.6 & 0.5 & 0.6 & 0.5 & 0.5 & 0.5 & 0.4 & 0.5 & 0.5 & 0.6 & 0.4 & 0.3 & 0.5 \\
\hline$M A-R A$ & 1.4 & 1.7 & 1.4 & 1.4 & 1.5 & 1.5 & 2.1 & 2.1 & 2.4 & 2.0 & 1.9 & 1.6 & 1.6 & 2.3 \\
\hline MA-human & 1 & 1 & 1 & 1 & 1 & 1 & 1 & 1 & 1 & 1 & 1 & 1 & 1 & 1 \\
\hline
\end{tabular}

The total number of rearrangements and the total number of interchromosomal rearrangements on the edges of the evolutionary tree between the human, mouse, rat, and chicken genomes (see top of Fig. 2) are shown for both gene-based and sequence-based data at various thresholds. MA is the mammalian ancestor and $R A$ is the rodent ancestor. The numbers are normalized using the MA-human edge to simplify comparison.

the path from human to chicken (132) is only slightly higher than the number of interchromosomal rearrangements on the path from human to mouse and rat (124 and 116). It implies an extremely slow rate of interchromosomal rearrangements along the chicken edge in the evolutionary tree: 0.19 rearrangements per million years on the $M A$-chicken edge as compared to 0.34 on the $M A$-human edge and 1.1 on the $M A$-mouse edge. ${ }^{8}$

${ }^{8} \mathrm{We}$ are using estimated divergence times of 16 milion years ago (Mya) for RA and 87 Mya for MA (Springer et al. 2003) and 310 Mya for birds and mammals (Hedges and Kumar 2004; Reisz and Muller 2004).

Table 3. Chromosome associations and syntenies of the reconstructed mammalian ancestor

\begin{tabular}{|c|c|c|c|c|c|c|c|}
\hline \multirow[b]{2}{*}{ Run } & \multicolumn{4}{|c|}{ Gene-based } & \multicolumn{3}{|c|}{ Sequence-based } \\
\hline & gene4 & gene7 & gene 10 & gene20 & $100 \mathrm{~K}$ & $200 \mathrm{~K}$ & $300 \mathrm{~K}$ \\
\hline \multicolumn{8}{|c|}{ Human chromosome associations in $M A$} \\
\hline $3 / 21$ & + & + & + & + & + & + & + \\
\hline $4 / 8$ & + & + & + & + & + & + & + \\
\hline $12 / 22 a$ & + & + & + & + & + & + & + \\
\hline $12 / 22 b$ & + & + & + & + & + & + & + \\
\hline $7 / 16$ & - & - & - & - & - & - & - \\
\hline $14 / 15$ & + & - & - & + & - & + & - \\
\hline $16 / 19$ & + & - & - & - & + & + & - \\
\hline \multicolumn{8}{|c|}{ Human chromosomes syntenies in $M A$} \\
\hline 8 & - & - & - & + & - & - & - \\
\hline 9 & - & - & - & - & - & + & - \\
\hline 13 & + & + & + & + & + & + & + \\
\hline 14 & + & + & + & + & + & + & + \\
\hline 15 & - & - & - & - & - & - & + \\
\hline 16 & - & - & + & + & - & - & - \\
\hline 17 & + & + & - & - & - & - & - \\
\hline 18 & - & - & - & + & - & - & - \\
\hline 19 & - & - & + & - & - & - & - \\
\hline 20 & + & + & + & + & + & + & + \\
\hline 21 & + & + & + & + & + & + & + \\
\hline$x$ & + & + & + & + & + & + & + \\
\hline \multicolumn{8}{|c|}{ Chicken chromosome syntenies in $M A$} \\
\hline 7 & - & - & - & - & + & - & - \\
\hline 10 & - & - & - & - & - & + & + \\
\hline 12 & - & + & - & - & - & - & - \\
\hline 13 & - & - & - & - & - & + & + \\
\hline 15 & - & + & - & + & - & - & - \\
\hline 16 & + & + & + & + & N/A & N/A & N/A \\
\hline 17 & + & + & - & - & + & + & - \\
\hline 18 & + & + & + & + & - & - & + \\
\hline 20 & + & + & + & + & + & + & + \\
\hline 21 & + & + & + & + & + & + & + \\
\hline 22 & - & - & - & - & - & - & + \\
\hline 23 & + & + & + & + & + & + & + \\
\hline 24 & + & + & + & + & + & + & + \\
\hline 27 & + & + & + & + & + & + & + \\
\hline 28 & - & - & + & - & - & - & - \\
\hline 32 & - & - & - & - & + & + & + \\
\hline W & - & - & - & - & - & - & - \\
\hline
\end{tabular}




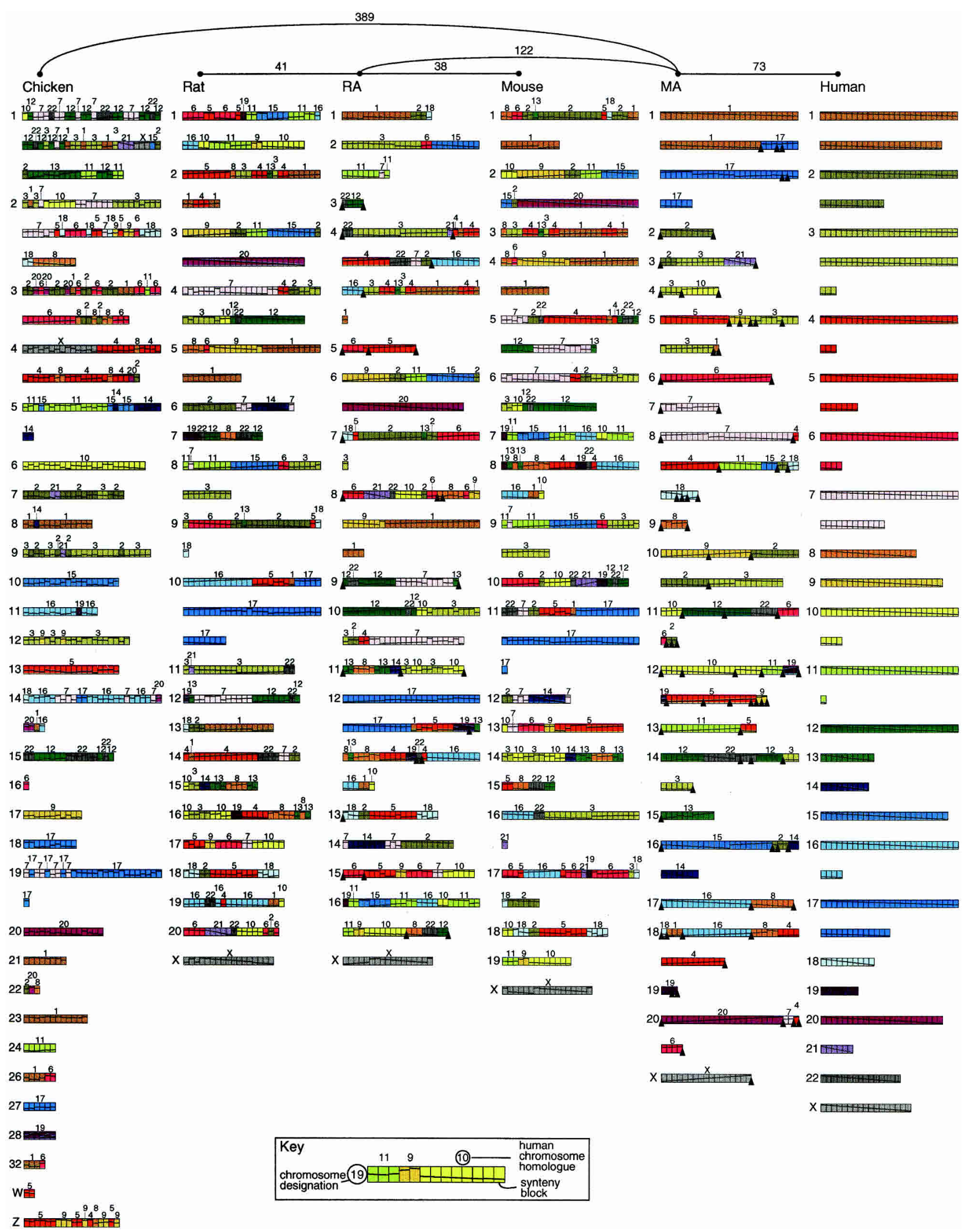

Figure 2. Ancestral murid rodent ancestor $(R A)$, ancestral mammalian ancestor $(M A)$, and evolutionary tree recovered by $M G R$ using the human, mouse, rat, and chicken genomes. Each genome is represented as an arrangement of 586 synteny blocks computed by GRIMM-Synteny on gene-based data in run gene7. Each synteny block is drawn as one unit, regardless of its length in nucleotides. Chromosomes with too many blocks are split into multiple lines. Each human chromosome is assigned a unique color, and a diagonal line is drawn through the whole chromosome. In other genomes, this diagonal line indicates the relative order and orientation of the rearranged blocks. Black triangles below ancestral chromosomes indicate weak adjacencies. The unrooted phylogram at the top of the figure shows the topology of the evolutionary tree; the last common ancestor of human, mouse, rat, and chicken would be somewhere on the edge between Chicken and MA. The minimum number of rearrangements required to convert between two genomes is shown on each edge of that tree. 
Figure 2 also reveals a large number of inversions that scramble the genomic make-up of individual chromosomes. For example, chicken Chromosome 19 (GGA19) is "built" from synteny blocks residing on only two human chromosomes (HSA7 and HSA17) that form a complex shuffle represented by eight different unicolored segments (four from HSA7, alternating with four from HSA17, and those in turn contain intrachromosomal rearrangements). These segments likely arose from a translocation of HSA7 and HSA17, creating a chromosome that was further shuffled by inversions on the evolutionary path between human and chicken. To sort out some of the intrachromosomal (inversions) from the interchromosomal (translocations/fusions/ fissions) rearrangements and in an attempt to "reverse" history, we perform a maximal number of initial inversions in the four starting genomes, ${ }^{9}$ thus making every chromosome less shuffled than it appears in Figure 2. By performing all these initial inversions, we reduce the number of four-way blocks from 586 down to 311 new "pre-ancestral" synteny blocks. We call these four modified genomes "pre-ancestors." ${ }^{10}$ Figure 3 illustrates the proposed organization of these pre-ancestors and of the two ancestors ( $R A$ and $M A$ ) using this smaller number of segments.

Because the pairwise distances between the initial genomes are substantial, it is possible to find alternative ancestors also minimizing the total number of rearrangement events on the evolutionary tree. By exploring some of these alternative ancestors (see Methods), we can partition all the adjacencies of the recovered ancestor into "strong" and "weak" adjacencies depending on whether they are present or not present in all of the observed alternative ancestors. ${ }^{11}$ In run gene7, we find 524 strong adjacencies and 83 weak adjacencies ${ }^{12}$ (see Fig. 2). Many of the previously postulated chromosome associations of the placental ancestor correspond to strong adjacencies in $M A$. These associations are $3 / 21,4 / 8,12 / 22 \mathrm{a}$, and $12 / 22 \mathrm{~b}$ (Murphy et al. 2003; Stanyon et al. 2003). Other ancestral chromosome associations missing from $M A$ (e.g., 7/16, 14/15, and 16/19) are currently only associated to other chromosomes by weak associations, meaning that in this analysis they were left as unresolved. The synteny of six human chromosomes $(13,14,17,20,21, \mathrm{X})$ and of 10 chicken chromosomes $(12,15-18,20,21,23,24,27)$ is preserved in MA. In stark contrast, the rodent Chromosome $\mathrm{X}$ is the only rodent chromosome whose synteny is preserved in $M A$. This is consistent with the affirmation that interchromosomal rearrangements have been very frequent in rodents. Results about chromosomal associations and syntenies are summarized in Table 3.

The reconstruction of the murid rodent ancestor, $R A$, is also coherent with the reconstruction of the same ancestor in Bourque et al. (2004). The human chromosome associations $3 / 21,4 / 8$, and $12 / 22$ are once again recovered in $R A$. The asso-

\footnotetext{
${ }^{9}$ The "maximal number of initial inversions" is the maximum we found, but there could be an alternative sequence with the same edge length and even more initial inversions.

${ }^{10}$ This definition of pre-ancestor differs from the one in Murphy et al. (2003), because the rearrangements performed are only inversions and we use the information about the reconstructed ancestors instead of the notion of "good rearrangement" (Bourque and Pevzner 2002).

${ }^{11}$ The number of weak adjacencies that we determine is actually a lower bound because we only explore a subset of all the alternative solutions.

${ }^{12}$ The total number of adjacencies is the total number of blocks plus the total number of chromosomes. Adjacencies include both "internal adjacencies" (blocks adjacent within a chromosome) and "external adjacencies" (blocks adjacent to a chromosome end).
}

ciation $16 / 19$ is missing from the current reconstruction, but blocks from these two human chromosomes are found on the same chromosome in $R A$ (although they are not adjacent). The analysis of alternative ancestors reveals 576 strong adjacencies and only 26 weak adjacencies in $R A$ (see Fig. 2).

Some large regions of mammalian genomes are extremely well conserved across many species. The X-chromosome is one such example where the limited amount of exchange of genetic material with the other chromosomes (see Fig. 1) allows a detailed analysis of its rearrangement history. In the most parsimonious rearrangement scenario of the 17 synteny blocks on the $\mathrm{X}$-chromosome of human-mouse-rat and of the homologous blocks on chicken Chromosomes 1 and 4, there are 20 rearrangements in total. There are no rearrangements between human and $M A$ (i.e., human order is ancestral), 14 rearrangements (13 inversions and one fusion) between chicken and $M A$, two inversions between $M A$ and $R A$, one inversion between mouse and $R A$, and three inversions between rat and $R A$. Moreover, the scenario recovered is optimal and $M A$ and $R A$ are unique. For that optimal score, the number of steps on each edge is unique, but the specific order is not.

The set of blocks on the human X-chromosome is an example of a set of blocks that is not interrupted by any foreign block (a block outside that set) in any of the four genomes (although blocks can reside on multiple chromosomes in each genome). We call this a set of contiguous blocks. Large sets of contiguous blocks are interesting because they can be analyzed for rearrangements independently from the rest of the genome. HSA8p and HSA13 is an example of a well-conserved region, but, unfortunately, it does not form a set of contiguous block in run gene7 because the blocks from HSA8p are interrupted on GGA3 by blocks from HSA2 and on GGA4 by blocks from HSA4 (see Fig. 2). Blocks from HSA8p on GGA3-4 were probably interspersed with other blocks by a series of more recent inversions. Fortunately, we can undo these inversions by using the pre-ancestors (see Fig. 3A) where HSA8p and HSA13 do form a set of contiguous blocks. This specific region is shown in Figure 3B. The rearrangement scenario highlights how some chromosomes are well preserved in chicken but shuffled in rodent, whereas others are well conserved in rodent but more shuffled in chicken.

\section{Sequence-based data}

We also generated similarity blocks using four-way unique alignments as anchors. We include three runs, called 100K, 200K, and $300 \mathrm{~K}$ (see Table 4 and Supplemental material). In run $300 \mathrm{~K}$, two anchors are put into the same block if they are within $300 \mathrm{~kb}$ of each other in all genomes, and then resulting blocks smaller than $300 \mathrm{~kb}$ are deleted. The other runs are analogous. See the Methods section for more details. Running MGR on each set of fourway blocks generated at $100 \mathrm{~K}, 200 \mathrm{~K}$, and $300 \mathrm{~K}$ (see Fig. 4) produces rearrangement scenarios and putative ancestors whose features are summarized in Tables 2 and 3.

We find that there are about four to five times as many total rearrangements between chicken and $M A$ as between human and $M A$. Between the rodents and $M A$, there are about twice as many. For interchromosomal rearrangements, there are about three times as many between chicken and $M A$ as there are between human and $M A$, and there are also three times as many between each rodent and $M A$ as between human and $M A$.

Some of the putative ancestral human chromosome associations were observed in all runs $(3 / 21,4 / 8,12 / 22 a$, and 12/22b), 


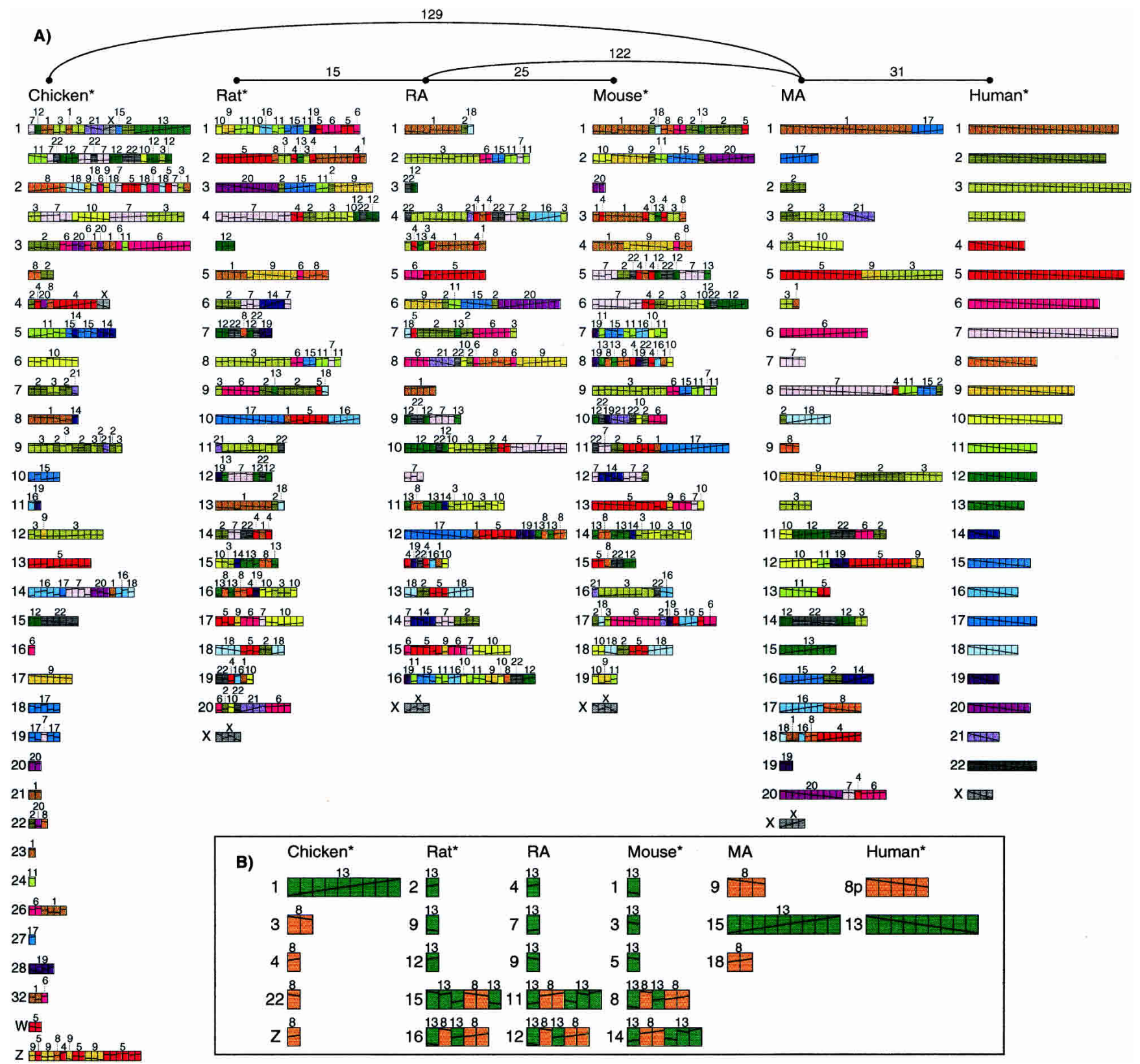

Figure 3. (A) Four pre-ancestors (marked with *), obtained after carrying out a maximal number of initial inversions, and two putative ancestors (murid rodent ancestor, $R A$, and mammalian ancestor, $M A$ ) for gene-based data in run gene7. Each genome is represented by an arrangement of 311 new synteny blocks obtained after carrying out the initial inversions and condensing the 586 initial four-way blocks. (B) Arrangement of the set of 14 blocks from HSA8p and HSA13, which are contiguous in the pre-ancestors of all species but are found on four different chromosomes in chicken, mouse, and rat. The two putative ancestral arrangements of these blocks, as recovered by MGR, are also shown.

while others were only recovered in some of the runs $(14 / 15$, 16/19) or not recovered at all (7/16). Some human chromosome syntenies were systematically preserved in $M A(13,14,20,21$, and $\mathrm{X}$ ) and similarly, some chicken chromosome syntenies were found in $M A$ for all runs $(20,21,23,24$, and 27). Many of the discrepancies observed in Table 3 can be explained by differences in the coverage of gene-based data versus sequence-based data. For instance, two chicken chromosome syntenies $(22,32)$ are only observed in sequence-based data mainly because they are covered by fewer blocks (1 block each in run 300K) (see Fig. 4) in those data sets. Chicken Chromosome 16 is an even more drastic example: in gene-based data a single short block represents it, whereas in sequence-based data, it is not represented at all.

\section{Microrearrangement scenarios}

The analysis of rearrangements in mammalian genomes is complicated by microrearrangements (small-scale rearrangements within synteny blocks). The GRIMM-Synteny algorithm preserves information about microrearrangements in each block it forms, allowing us to run MGR independently on the genes or anchors within each synteny block. This generated a microrear-

\section{Genome Research}

www.genome.org 
Table 4. Four-way human-mouse-rat-chicken synteny blocks based on sequence alignments

\begin{tabular}{|c|c|c|c|c|}
\hline & Human & Mouse & Rat & Chicken \\
\hline $\begin{array}{l}\text { Chromosomes included } \\
\text { Total length }(\mathrm{Mb})\end{array}$ & $\begin{array}{c}1-22, x \\
3020\end{array}$ & $\begin{array}{l}1-19, x \\
2577\end{array}$ & $\begin{array}{c}1-20, x \\
2720\end{array}$ & $\begin{array}{c}1-24,26-28,32, W, Z \\
940\end{array}$ \\
\hline $\begin{array}{l}\text { Run } 100 \mathrm{~K} \\
\text { No. of blocks } \\
\text { Mean synteny block length }(\mathrm{kb}) \\
\text { Max synteny block length }(\mathrm{kb}) \\
\text { Total synteny block length }(\mathrm{kb}) \\
\text { Total synteny block length (\% of genome) } \\
\text { No. of breakpoint regions } \\
\text { Mean breakpoint region length }(\mathrm{kb}) \\
\text { Max breakpoint region length }(\mathrm{kb}) \\
\text { Total breakpoint region length (kb) } \\
\text { Total breakpoint region length (\% of genome) } \\
\text { No. of anchors per block }\end{array}$ & $\begin{array}{c}583 \\
2713 \\
44,708 \\
1,581,616 \\
52.37 \% \\
560 \\
2254 \\
40,507 \\
1,262,211 \\
41.80 \% \\
5-2915, \text { mean } 234.2\end{array}$ & $\begin{array}{c}583 \\
2436 \\
38,350 \\
1,420,395 \\
55.11 \% \\
563 \\
1815 \\
19,233 \\
1,021,678 \\
39.64 \%\end{array}$ & $\begin{array}{c}583 \\
2566 \\
41,531 \\
1,495,964 \\
55.00 \% \\
562 \\
2065 \\
33,229 \\
1,160,670 \\
42.67 \%\end{array}$ & $\begin{array}{c}583 \\
1083 \\
16,085 \\
631,341 \\
67.15 \% \\
553 \\
509 \\
5247 \\
281,338 \\
29.92 \%\end{array}$ \\
\hline $\begin{array}{l}\text { Run } 200 \mathrm{~K} \\
\text { No. of blocks } \\
\text { Mean synteny block length }(\mathrm{kb}) \\
\text { Max synteny block length }(\mathrm{kb}) \\
\text { Total synteny block length }(\mathrm{kb}) \\
\text { Total synteny block length }(\% \text { of genome) } \\
\text { No. of breakpoint regions } \\
\text { Mean breakpoint region length }(\mathrm{kb}) \\
\text { Max breakpoint region length }(\mathrm{kb}) \\
\text { Total breakpoint region length }(\mathrm{kb}) \\
\text { Total breakpoint region length (\% of genome) } \\
\text { No. of anchors per block }\end{array}$ & $\begin{array}{c}474 \\
3556 \\
44,292 \\
1,685,370 \\
55.81 \% \\
451 \\
2534 \\
46,565 \\
1,142,700 \\
37.84 \% \\
11-3213, \text { mean } 319.6\end{array}$ & $\begin{array}{c}474 \\
3191 \\
38,350 \\
1,512,364 \\
58.68 \% \\
454 \\
2034 \\
14,435 \\
923,594 \\
35.84 \%\end{array}$ & $\begin{array}{c}474 \\
3365 \\
41,501 \\
1,595,170 \\
58.65 \% \\
453 \\
2316 \\
32,219 \\
1,049,169 \\
38.57 \%\end{array}$ & $\begin{array}{c}474 \\
1409 \\
16,085 \\
667,927 \\
71.04 \% \\
444 \\
550 \\
7563 \\
244,138 \\
25.97 \%\end{array}$ \\
\hline $\begin{array}{l}\text { Run } 300 \mathrm{~K} \\
\text { No. of blocks } \\
\text { Mean synteny block length }(\mathrm{kb}) \\
\text { Max synteny block length }(\mathrm{kb}) \\
\text { Total synteny block length }(\mathrm{kb}) \\
\text { Total synteny block length }(\% \text { of genome) } \\
\text { No. of breakpoint regions } \\
\text { Mean breakpoint region length }(\mathrm{kb}) \\
\text { Max breakpoint region length }(\mathrm{kb}) \\
\text { Total breakpoint region length }(\mathrm{kb}) \\
\text { Total breakpoint region length }(\% \text { of genome) } \\
\text { No. of anchors per block }\end{array}$ & $\begin{array}{c}430 \\
4049 \\
45,607 \\
1,740,889 \\
57.65 \% \\
407 \\
2662 \\
44,269 \\
1,083,594 \\
35.88 \% \\
16-3410, \text { mean } 370.6\end{array}$ & $\begin{array}{c}430 \\
3620 \\
38,350 \\
1,556,569 \\
60.40 \% \\
410 \\
2136 \\
15,587 \\
875,902 \\
33.99 \%\end{array}$ & $\begin{array}{c}430 \\
3814 \\
42,466 \\
1,639,876 \\
60.29 \% \\
409 \\
2451 \\
32,219 \\
1,002,423 \\
36.85 \%\end{array}$ & $\begin{array}{c}430 \\
1596 \\
16,085 \\
686,262 \\
72.99 \% \\
400 \\
562 \\
10,187 \\
224,812 \\
23.91 \%\end{array}$ \\
\hline
\end{tabular}

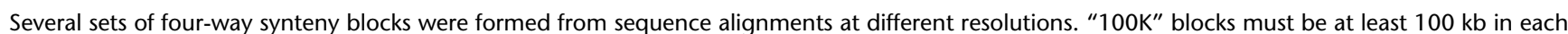
species and allow for anchors to be displaced up to $100 \mathrm{~kb}$ in a certain metric, as explained in the text under "GRIMM-Synteny parameters." Statistics are shown on the sizes of the blocks and the breakpoint regions between them (excluding chromosome ends).

rangement scenario, including a microevolutionary tree, for each of these synteny blocks. See Figure 5 for an example. We looked at the usefulness of using microrearrangements as evolutionary characters for phylogenetic tree reconstructions. A microevolutionary tree is said to be a "perfect match" if its topology agrees with the known topology for human-mouse-rat-chicken (as depicted at the top of Fig. 2). ${ }^{13}$ If it does not agree with the known topology, it is said to be a mismatch. A recovered topology can only agree or disagree with the known topology if the internal edge of the recovered tree has at least one rearrangement. Otherwise, the recovered topology is said to be inconclusive. The results are summarized in Table 5 . The trees recovered are very accurate: for both gene-based and sequence-based data, the proportion of mismatches is very low. For gene-based data, a large proportion of synteny blocks $(\sim 65 \%)$ do not have any microrearrangements, which leads to inconclusive topologies. In contrast, with sequence-based data, this proportion is

\footnotetext{
${ }^{13} \mathrm{~A}$ topology is said to agree with another if the sets of partitions of leaves
} defined by the internal edges with at least one rearrangement are the same. relatively small ( 25\%) and leads to a higher fraction of perfect matches $(\sim 25 \%)$. As expected, the total number of microrearrangements is much higher for sequence-based data than for gene-based data. This is explained in part by the fact that many of the microrearrangements in sequence-based synteny blocks lay outside exons.

We separately ran MGR within the same synteny blocks while imposing the known topology for human-mouse-ratchicken; this only leads to a slight increase in the total number of microrearrangements (see Table 5).

In Table 6 , we compute the proportion of the microrearrangements over the different edges of the tree. The results show that most of the microrearrangements $(\sim 60 \%)$ occurred on the chicken to $M A$ edge but that a large portion $(-20 \%)$ occurred on the rat to $M A$ edge. If we take into account the divergence time, this suggests either an accelerated rate of microrearrangements in rat or a higher rate of errors in the rat assembly. Figure 5 shows a block in which a large inversion occurred on the evolutionary path leading to rodents but in which an additional six inversions are required to explain the arrangement of anchors in rat. 


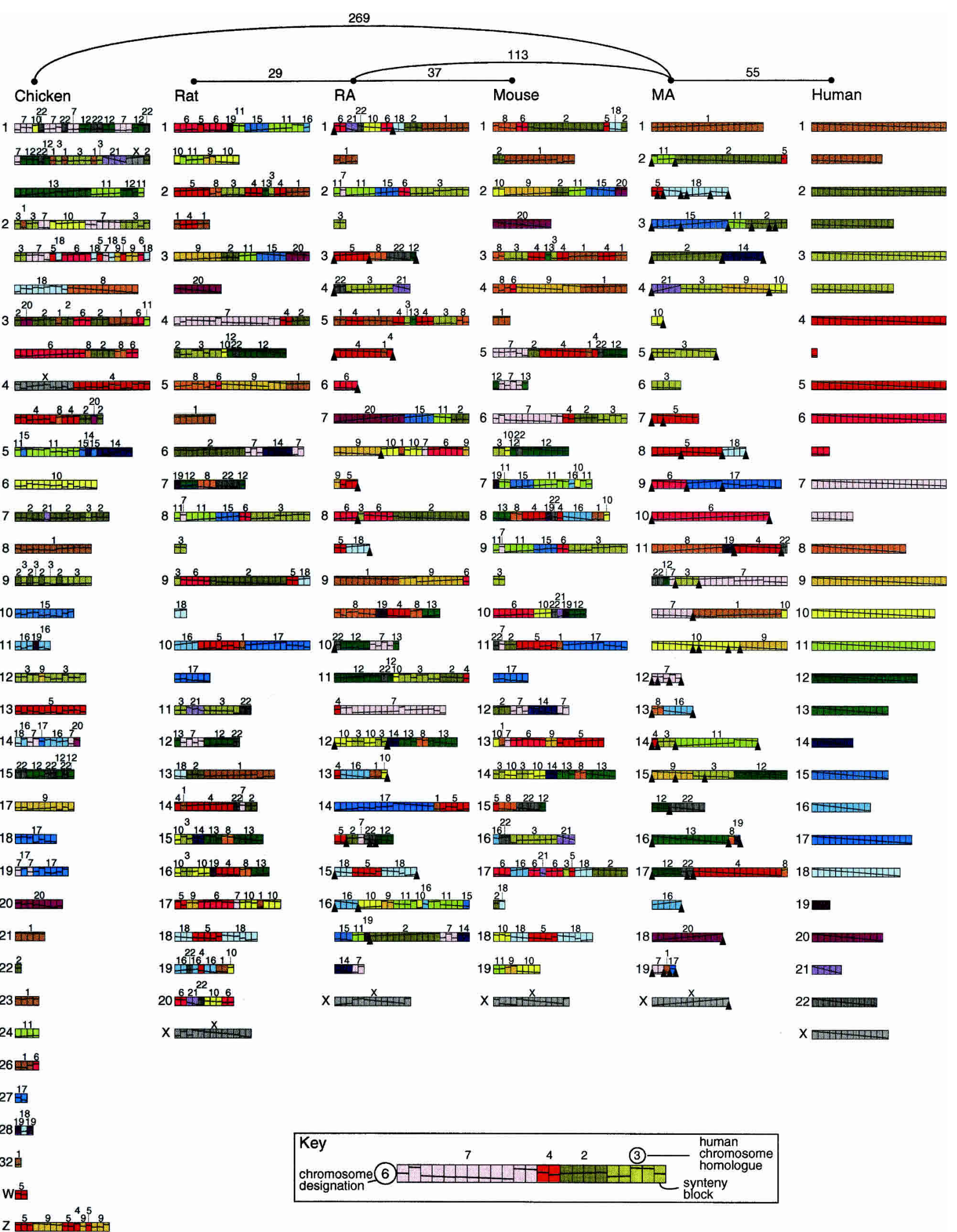

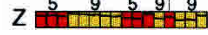

Figure 4. Ancestral murid rodent ancestor $(R A)$, ancestral mammalian ancestor $(M A)$, and evolutionary tree recovered by MGR using the human, mouse, rat, and chicken genomes. Each genome is represented as an arrangement of 430 synteny blocks computed by GRIMM-Synteny on sequencebased data in run $300 \mathrm{~K}$. 

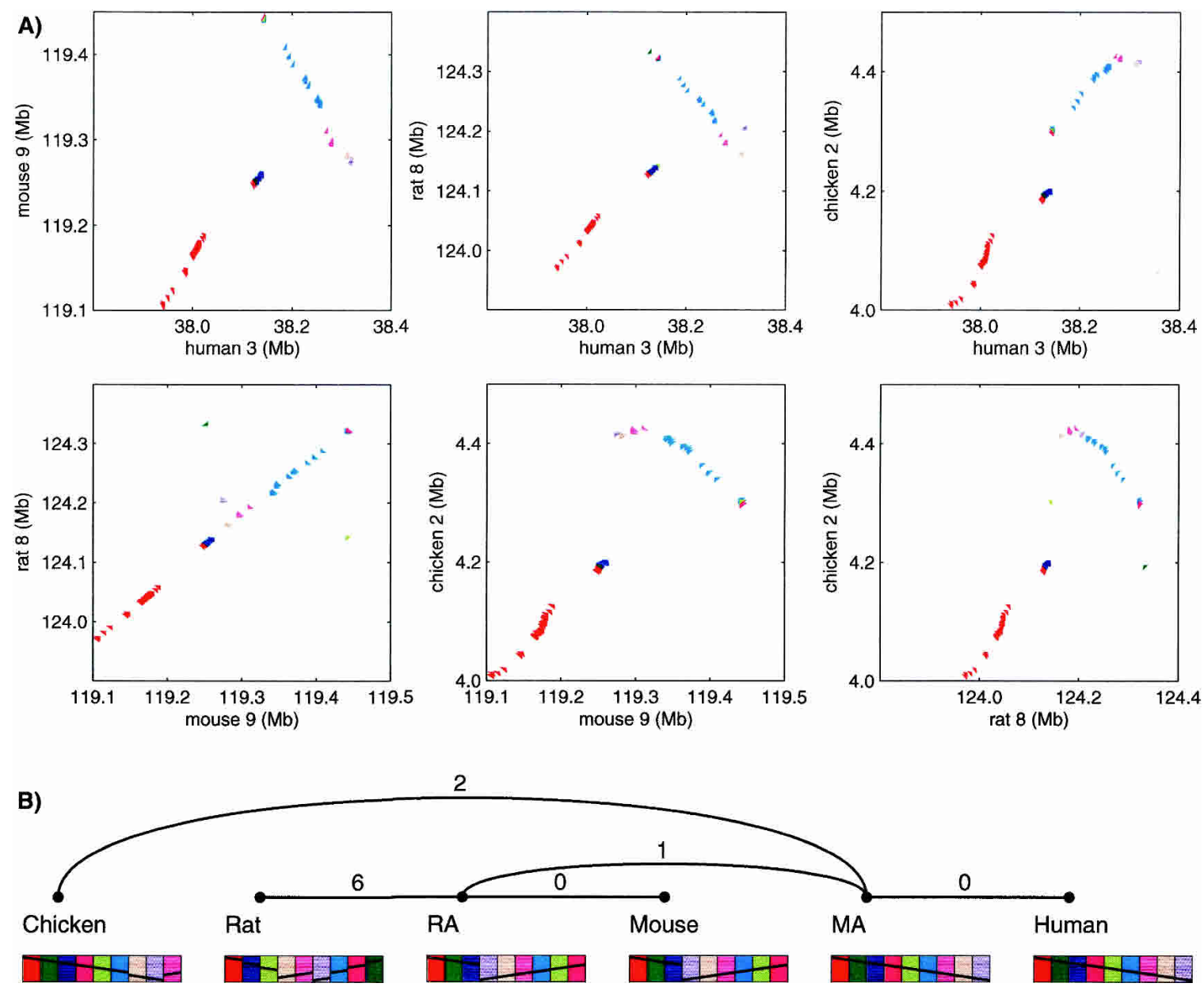

Figure 5. (A) Dot-plots of similarity anchors in a four-way synteny block with microrearrangements. The block was computed by GRIMM-Synteny on sequence-based data in run $300 \mathrm{~K}$. The block length is $380 \mathrm{~kb}$ in human, $340 \mathrm{~kb}$ in mouse, $364 \mathrm{~kb}$ in rat, and $419 \mathrm{~kb}$ in chicken. The anchors divide into nine groups, indicated by different colors; these groups have a different order and orientation in each genome. Within each group, the anchors have the same relative order in all genomes (or an overall flip of the whole group), but their absolute spacing is not preserved. (B) Microrearrangement history for the same block as generated by MGR. The nine colored blocks correspond to the nine groups of anchors in the dot-plots. The minimum number of inversions required to convert between synteny blocks in two genomes is shown on each edge of the tree.

\section{Discussion}

Choosing a set of orthologous genes rather than the "similarity anchors" as in Gibbs et al. (2004) has some benefits. Since the chicken genome is rather distant from mammalian genomes, using similarity anchors (at lower similarity thresholds, due to the distance) leads to many spurious hits (Fig. 1), which are difficult to unambiguously assemble into synteny blocks. Moreover,

Table 5. Microrearrangements scenarios on applying MGR independently to genes/anchors within synteny blocks

\begin{tabular}{|c|c|c|c|c|c|c|c|}
\hline \multirow[b]{2}{*}{ Run } & \multicolumn{4}{|c|}{ Gene-based } & \multicolumn{3}{|c|}{ Sequence-based } \\
\hline & gene4 & gene7 & gene10 & gene20 & $100 \mathrm{~K}$ & $200 K$ & $300 \mathrm{~K}$ \\
\hline Blocks & 627 & 586 & 567 & 518 & 583 & 474 & 430 \\
\hline Conserved blocks & 0.758 & 0.660 & 0.628 & 0.577 & 0.425 & 0.259 & 0.193 \\
\hline Perfect match & 0.016 & 0.026 & 0.026 & 0.037 & 0.177 & 0.251 & 0.298 \\
\hline Mismatch & 0.002 & 0.007 & 0.009 & 0.010 & 0.003 & 0.006 & 0.016 \\
\hline Inconclusive & 0.982 & 0.967 & 0.965 & 0.953 & 0.820 & 0.743 & 0.686 \\
\hline Microrearrangements & 272 & 383 & 437 & 543 & 1408 & 1941 & 2329 \\
\hline Microrearrangements (force topology) & 273 & 387 & 442 & 548 & 1410 & 1944 & 2336 \\
\hline
\end{tabular}

"Blocks" indicates the number of synteny blocks in each run. "Conserved blocks" stands for the fraction of synteny blocks in which gene/anchor order is preserved in human-mouse-rat-chicken. "Perfect match" is the fraction of recovered topologies that agrees with the known topology for these four genomes (this requires the internal edge to have at least one rearrangement). "Mismatch" is the fraction of recovered topologies that do not agree with the known topology. "Inconclusive" is the fraction of recovered topologies with a zero-length internal edge, which cannot be used to verify any topology. "Microrearrangements" indicates the total number of rearrangements within these synteny blocks. "Microrearrangements (force topology)" is similar, but the scenario is computed with the constraint that it must obey the correct topology. 
Table 6. Estimated proportion of microrearrangements on each edge of the tree

\begin{tabular}{|c|c|c|c|c|c|c|c|}
\hline \multirow[b]{2}{*}{ Run } & \multicolumn{4}{|c|}{ Gene-based } & \multicolumn{3}{|c|}{ Sequence-based } \\
\hline & gene4 & gene7 & gene10 & gene20 & $100 \mathrm{~K}$ & $200 K$ & $300 K$ \\
\hline MA-chicken & 0.60 & 0.60 & 0.63 & 0.64 & 0.65 & 0.61 & 0.58 \\
\hline$R A$-mouse & 0.03 & 0.03 & 0.03 & 0.04 & 0.10 & 0.08 & 0.09 \\
\hline$R A$-rat & 0.23 & 0.22 & 0.20 & 0.17 & 0.14 & 0.18 & 0.20 \\
\hline$M A-R A$ & 0.04 & 0.05 & 0.06 & 0.05 & 0.09 & 0.09 & 0.09 \\
\hline MA-human & 0.09 & 0.10 & 0.10 & 0.10 & 0.03 & 0.3 & 0.03 \\
\hline
\end{tabular}

Scenarios were obtained by applying MGR independently to genes/ anchors within synteny blocks and imposing the proper topology shown at the top of Figure 2. Columns do not always add up to 1 because of rounding.

highly diverged genes may not generate any four-way anchors (based on exact nucleotide $l$-mer matches) but still can be reliably detected with amino acid scoring matrices.

Using GRIMM-Synteny on "similarity anchors" also has advantages. For instance, it allows avoiding the identification of orthologous versus paralogous copies of genes. It is also less affected by high-copy-number gene families (such as kinases or GPCRs). Moreover, it retains information in regions outside of exons. This extra information can be useful for the analysis of microrearrangements, but it can also be an asset in the reconstruction of the ancestral genomes by keeping stronger footprints of past events.

Although the two initial data sets are different, the properties of the evolutionary tree and of the reconstructed ancestors obtained at various thresholds are largely consistent. We observed a high ratio of inversions over all types of rearrangements in chicken but, overall, a relatively slow rate of rearrangements in this lineage. The large number of inversions in chicken can also be confirmed by the analysis microrearrangements within the synteny blocks. Finally, we also observed an accelerated rate of interchromosomal rearrangements in rodents.

Future developments could include more systematic ways of comparing synteny blocks generated using different programs, different sets of parameters, different types of input (e.g., gene vs. sequence data) but also different sets of initial genomes. Similarly, new metrics could also be developed to compare the ancestral reconstructions not only at the level of chromosome associations, chromosome syntenies, and rates of rearrangements but also at the level of actual synteny blocks and suggested adjacencies. Such metrics would need to account for the multiplicity of alternative solutions.

\section{Methods}

We compared these assemblies: Human (NCBI build 34, July 2003; UCSC hg16); Mouse (NCBI build 30, Feb. 2003; UCSC mm3); Rat (Baylor HGSC v. 3.1, June 2003; UCSC rn3); and Chicken (WUSTL Feb.2004, UCSC galGal2).

Gene-based comparisons used a set of 6447 four-way orthologous genes obtained by intersection of less strictly defined pairwise synteny maps, requiring at least two neighboring orthologous genes but allowing for up to four intervening genes, computed with SyntQL (Zdobnov et al. 2002; E. Zdobnov, unpubl.) on all best reciprocal BLASTP matches (Altschul et al. 1997) in cross-genome comparison. SyntQL relies on an optimized relational database engine to retrieve all minimal synteny blocks defined by a number of constraints (e.g., two pairs of orthologous genes between two genomes located closely on these genomes) formulated in an SQL query that are then merged into longer synteny blocks over shared orthologous pairs.

Sequence-based comparisons used alignments computed by Angie Hinrichs in the UCSC consortium using BLASTZ, MULTIZ, and other tools (Kent et al. 2003; Schwartz et al. 2003; Blanchette et al. 2004). Initially these contained 343,645 four-way alignments (and many more partial alignments, which we did not use); however, many of these four-way alignments have conflicting coordinates. We filtered out regions with duplications identified by Evan Eichler (in prep.) and other duplications we observed on dot-plots, and then applied the GRIMM-Anchors algorithm (Bourque et al. 2004) to reduce it to 208,032 four-way anchors.

\section{GRIMM-Synteny parameters}

We added more parameters to the GRIMM-Synteny algorithm, described for sequence-based data in Pevzner and Tesler (2003) and for unsigned gene-based data in Murphy et al. (2003). In the present study, for sequence-based data, we start with a set of anchors (four-way unique alignments in this case) described by their chromosome, start and end in nucleotides, and orientation. The distance between two points $\left(x_{1}, x_{2}, x_{3}, x_{4}\right)$ and $\left(y_{1}, y_{2}, y_{3}, y_{4}\right.$; measured in nucleotides) in the same four-way chromosome window is the Manhattan distance $\left|x_{1}-y_{1}\right|+\ldots+\left|x_{4}-y_{4}\right|$. The distance between two anchors is the distance between their closest terminals (there are two choices of terminal for each anchor, determined by the signs of the alignments). In the original GRIMM-Synteny, two anchors were joined together when the distance was less than a specified threshold. We now use a per species distance: the closest terminals are determined based on the total distance, and then the anchors are joined if in all species, the gap $\left|x_{i}-y_{i}\right|$ is (strictly) below the per species gap threshold $G_{i}$. The "300K" data set in the text of this paper uses $G_{i}=300,000$ for all species. The " $100 \mathrm{~K}$ " and " $200 \mathrm{~K}$ " data sets in the tables use $G_{i}=100,000$ and $G_{i}=200,000$, respectively.

In the original GRIMM-Synteny, we then discarded blocks whose span was below a minimum size in human. In this study, we set minimums in all species. In the present study, we discarded blocks whose span was (strictly) below a minimum size $C_{i}$ in any species; in the sequence-based runs $100 \mathrm{~K}, 200 \mathrm{~K}$, and $300 \mathrm{~K}$, we chose to set $C_{i}=G_{i}$. Occasionally, the blocks that make it past this filter will have conflicting coordinates in one species (the coordinate interval of block A is a subinterval of block B in one species), in which case we split the blocks up to resolve this.

We then merged together blocks that form a strip of consecutive blocks in the exact same order (allowing an overall flip) and chromosome window in all genomes, without interruption by other blocks. This step is appropriate for analyzing rearrangements, but may not be appropriate for other purposes.

Our "signed" gene-based data was analyzed with the same procedure but with a "gene" metric instead of a "nucleotide" metric; all genes in the data set are assigned an identical size. Specifically, the $j$-th consecutive gene in a chromosome is assigned span $2 j$ through $2 j+1$, and the orientation of the gene determines which coordinate is the "start" and which is the "end." Our "gene7" data set used throughout this paper uses the procedure described above with $G_{i}=7$ for all species. Two genes $\mathrm{A}$ and $\mathrm{B}$ are joined together if there are up to two intervening genes between them in every species, with certain constraints on flipping A and B: at two intervening genes, the relative orientations of $A$ and $B$ must be the same in all species or one of them can be flipped, and with less than two intervening genes, either

\section{Genome Research}

www.genome.org 
or both can be flipped. Next, we discarded blocks supported by less than three genes, and finally, we merged together strips of blocks as before. The gene 4 , gene 10 , and gene 20 data sets similarly used 4,10 , or 20 in place of $7 . G_{i}=4$ (respectively, 10 or 20) has the effect of allowing 1 (respectively, 4 or 9) intervening gene, but A and B must remain in the same relative orientation in all species, or up to 0 (respectively, 3 or 8 ) intervening genes, but $\mathrm{A}$ and $\mathrm{B}$ may be flipped arbitrarily.

In contrast, the data set in Murphy et al. (2003) was "unsigned" gene-based data, because some data were obtained from $\mathrm{RH}$ maps and therefore signs were not available. All genes were given equal size 1 , the $j$-th gene on a chromosome was assigned the single point coordinate $j$, and had just one end instead of two.

\section{Search for alternative ancestors}

Starting from the ancestral reconstruction MA obtained with MGR and the three adjacent genomes on the evolutionary tree (human, chicken, and $R A$ ), we generated a list of rearrangements in $M A$ that did not increase the sum of the distances to those adjacent genomes. An alternative ancestor (at distance 1 from $M A)$ is associated to each of these rearrangements. We repeated the process starting from these new alternative ancestors by using both a breadth-first-search and a depth-first-search approach. We kept the first 6000 alternative ancestors (3000 from each approach). We used a similar method to look for alternative reconstructions of $R A$.

Specifically, in run gene7, we found a list of 83 rearrangements in $M A$ that did not increase the overall tree score. Using a breadth-first-search approach, we looked at all 83 corresponding alternative ancestors (at distance 1 from $M A$ ). We generated a new list of rearrangements that did not increase the overall tree score for each of these alternative ancestors. Since most of the rearrangements from the initial list of 83 are commutative, the number of alternative ancestors at distance 2 from $M A$ is $\sim(83 \times 82) / 2=3403$. In practice, it could even be larger because some of these rearrangements "unlock" new rearrangements that do not increase the overall score either. For the purpose of the current analysis, we stopped once we had a total of 3000 distinct alternative ancestors (83 at distance 1 from MA and 2917 at distance 2).

We also used a depth-first-search approach to look for different alternative ancestors of $M A$. Starting only from the first alternative ancestor identified at distance 1 from $M A$, we found 80 alternative ancestors at distance 2 . Starting from the first of these alternative ancestors, we found 80 new alternative ancestors but at distance 3 from $M A$. We repeated the process iteratively. When the first ancestor at distance $x$ did not suggest any ancestor at distance $x+1$, we moved down to another ancestor at distance $x$. When we ran out of ancestors at distance $x$, we stepped back to the list of ancestors at distance $x-1$. In practice, we stopped once we reached a total of 3000 distinct ancestors found at various distances from $M A$. In run gene7, the distance between $M A$ and the alternative ancestors found with the depthfirst-search approach ranged from 1 to 38 rearrangements.

\section{Acknowledgments}

We are grateful to LaDeanna Hillier, Ross Hardison, Bill Murphy, Lior Pachter, and Angie Hinrichs for many helpful discussions and suggestions. We also thank Angie Hinrichs for providing the sequence-based alignments and the anonymous reviewers for valuable recommendations. G.B. is supported by a fellowship of the Fonds Québecois de la Recherche sur la Nature et les Technologies.

\section{References}

Altschul, S.F., Madden, T.L., Schaffer, A.A., Zhang, J., Zhang, Z., Miller W., and Lipman, D.J. 1997. Gapped BLAST and PSI-BLAST: A new generation of protein database search programs. Nucleic Acids Res. 25: 3389-3402.

Bafna, V. and Pevzner, P. 1995. Sorting by reversals: Genome rearrangements in plant organelles and evolutionary history of $\mathrm{X}$ chromosome. Mol. Biol. Evol. 12: 239-246.

Blanchette, M., Kunisawa, T., and Sankoff, D. 1999. Gene order breakpoint evidence in animal mitochondrial phylogeny. J. Mol. Evol. 49: 193-203.

Blanchette, M., Kent, W.J., Riemer, C., Elnitski, L., Smit, A.F., Roskin, K.M., Baertsch, R., Rosenbloom, K., Clawson, H., Green, E.D., et al. 2004. Aligning multiple genomic sequences with the threaded blockset aligner. Genome Res. 14: 708-715.

Bourque, G. and Pevzner, P.A. 2002. Genome-scale evolution: Reconstructing gene orders in the ancestral species. Genome Res. 12: $26-36$.

Bourque, G., Pevzner, P.A., and Tesler, G. 2004. Reconstructing the genomic architecture of ancestral mammals: Lessons from human, mouse, and rat genomes. Genome Res. 14: 507-516.

Cosner, M.E., Jansen, R.K., Moret, B.M., Raubeson, L.A., Wang, L.S., Warnow, T., and Wyman, S. 2000. A new fast heuristic for computing the breakpoint phylogeny and experimental phylogenetic analyses of real and synthetic data. Proc. Int. Conf. Intell. Syst. Mol. Biol. 8: 104-115.

Gibbs, R.A., Weinstock, G.M., Metzker, M.L., Muzny, D.M., Sodergren, E.J., Scherer, S., Scott, G., Steffen, D., Worley, K.C., Burch, P.E., et al 2004. Genome sequence of the Brown Norway rat yields insights into mammalian evolution. Nature 428: 493-521.

Hannenhalli, S. and Pevzner, P. 1995. Transforming men into mice: Polynomial algorithm for genomic distance problem. Thirty-Sixth IEEE Symposium on Foundations of Computer Science, pp. 581-592. IEEE Press, Los Alamos, CA.

Hedges, S.B. and Kumar, S. 2004. Precision of molecular time estimates. Trends Genet. 20: 242-247.

Kent, W.J., Baertsch, R., Hinrichs, A., Miller, W., and Haussler, D. 2003. Evolution's cauldron: Duplication, deletion, and rearrangement in the mouse and human genomes. Proc. Natl. Acad. Sci. 100: $11484-11489$

Lander, E.S., Linton, L.M., Birren, B., Nusbaum, C., Zody, M.C., Baldwin, J., Devon, K., Dewar, K., Doyle, M., FitzHugh, W., et al. 2001. Initial sequencing and analysis of the human genome. Nature 409: 860-921.

Larget, B., Simon, D.L., and Kadane, B.J. 2002. Bayesian phylogenetic inference from animal mitochondrial genome arrangements. J. Roy. Stat. Soc. B 64: 681-695.

Murphy, W.J., Sun, S., Chen, Z., Yuhki, N., Hirschmann, D., Menotti-Raymond, M., and O'Brien, S.J. 2000. A radiation hybrid map of the cat genome: Implications for comparative mapping. Genome Res. 10: 691-702.

Murphy, W.J., Eizirik, E., O’Brien, S.J., Madsen, O., Scally, M., Douady, C.J., Teeling, E., Ryder, O.A., Stanhope, M.J., de Jong, W.W., et al. 2001. Resolution of the early placental mammal radiation using Bayesian phylogenetics. Science 294: 2348-2351.

Murphy, W.J., Bourque, G., Tesler, G., Pevzner, P., and O'Brien, S.J. 2003. Reconstructing the genomic architecture of mammalian ancestors using multispecies comparative maps. Hum. Genom. 1: $30-40$.

O'Brien, S.J., Menotti-Raymond, M., Murphy, W.J., Nash, W.G., Wienberg, J., Stanyon, R., Copeland, N.G., Jenkins, N.A., Womack, J.E., and Marshall Graves, J.A. 1999. The promise of comparative genomics in mammals. Science 286: 458-462, 479-481.

Palmer, J.D. and Herbon, L.A. 1988. Plant mitochondrial DNA evolves rapidly in structure, but slowly in sequence. J. Mol. Evol. 28: 87-97.

Pevzner, P. and Tesler, G. 2003. Genome rearrangements in mammalian evolution: Lessons from human and mouse genomes. Genome Res. 13: $37-45$.

Reisz, R.R. and Muller, J. 2004. Molecular timescales and the fossil record: A paleontological perspective. Trends Genet. 20: 237-241.

Sankoff, D., Leduc, G., Antoine, N., Paquin, B., Lang, B.F., and Cedergren, R. 1992. Gene order comparisons for phylogenetic inference: Evolution of the mitochondrial genome. Proc. Natl. Acad. Sci. 89: 6575-6579.

Schwartz, S., Kent, W.J., Smit, A., Zhang, Z., Baertsch, R., Hardison, R.C., Haussler, D., and Miller, W. 2003. Human-mouse alignments with BLASTZ. Genome Res. 13: 103-107.

Springer, M.S., Murphy, W.J., Eizirik, E., and O'Brien, S.J. 2003. Placental mammal diversification and the Cretaceous-Tertiary 


\section{Bourque et al.}

boundary. Proc. Natl. Acad. Sci. 100: 1056-1061.

Stanyon, R., Stone, G., Garcia, M., and Froenicke, L. 2003. Reciprocal chromosome painting shows that squirrels, unlike murid rodents, have a highly conserved genome organization. Genomics 82: $245-249$

Tesler, G. 2002a. Efficient algorithms for multichromosomal genome rearrangements. J. Comp. Sys. Sci. 65: 587-609. - 2002b. GRIMM: Genome rearrangements web server. Bioinformatics 18: 492-493.

Waterston, R.H., Lindblad-Toh, K., Birney, E., Rogers, J., Abril, J.F., Agarwal, P., Agarwala, R., Ainscough, R., Alexandersson, M., An, P., et al. 2002. Initial sequencing and comparative analysis of the mouse genome. Nature 420: 520-562.

Zdobnov, E.M., von Mering, C., Letunic, I., Torrents, D., Suyama, M, Copley, R.R., Christophides, G.K., Thomasova, D., Holt, R.A., Subramanian, G.M., et al. 2002. Comparative genome and proteome analysis of Anopheles gambiae and Drosophila melanogaster. Science 298: $149-159$.

Received July 14, 2004; accepted in revised form October 4, 2004. 


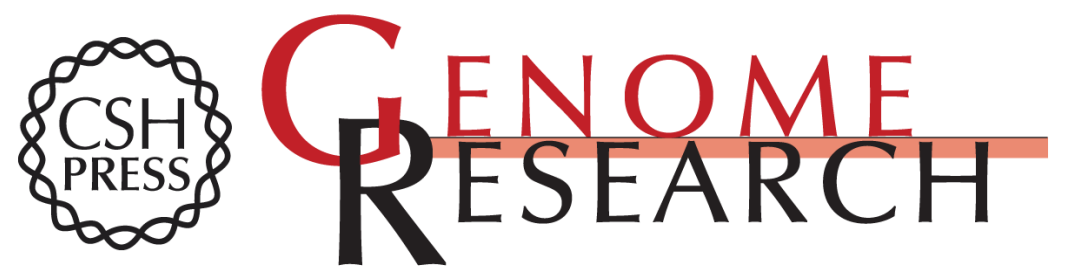

\section{Comparative architectures of mammalian and chicken genomes reveal highly variable rates of genomic rearrangements across different lineages}

Guillaume Bourque, Evgeny M. Zdobnov, Peer Bork, et al.

Genome Res. 2005 15: 98-110

Access the most recent version at doi:10.1101/gr.3002305

$\begin{array}{r}\text { Supplemental } \\ \text { Material }\end{array}$ http://genome.cshlp.org/content/suppl/2004/12/08/gr.3002305.DC1
htpeme.cshlp.org/content/suppl/2004/12/10/gr.3002305.DC2

References This article cites 27 articles, 12 of which can be accessed free at:

http://genome.cshlp.org/content/15/1/98.full.html\#ref-list-1

\section{License}

Email Alerting

Receive free email alerts when new articles cite this article - sign up in the box at the Service top right corner of the article or click here.

\section{Affordable, Accurate Sequencing.}

